\title{
Genome-wide variation and identification of vaccine targets in the Plasmodium falciparum genome
}

\author{
Jianbing $\mathrm{Mu}^{1}$, Philip Awadalla ${ }^{2}$, Junhui Duan ${ }^{1}$, Kate M McGee ${ }^{2}$, Jon Keebler ${ }^{2}$, Karl Seydel ${ }^{1}$, \\ Gilean A T McVean ${ }^{3}$ \& Xin-zhuan $\mathrm{Su}^{1}$
}

One goal in sequencing the Plasmodium falciparum genome, the agent of the most lethal form of malaria, is to discover vaccine and drug targets ${ }^{1}$. However, identifying those targets in a genome in which $\sim 60 \%$ of genes have unknown functions is an enormous challenge. Because the majority of known malaria antigens and drug-resistant genes are highly polymorphic and under various selective pressures ${ }^{2-6}$, genome-wide analysis for signatures of selection may lead to discovery of new vaccine and drug candidates. Here we surveyed 3,539 $P$. falciparum genes ( $\sim 65 \%$ of the predicted genes) for polymorphisms and identified various highly polymorphic loci and genes, some of which encode new antigens that we confirmed using human immune sera. Our collections of genome-wide SNPs $(\sim 65 \%$ nonsynonymous) and polymorphic microsatellites and indels provide a high-resolution map (one marker per $\sim 4 \mathrm{~kb}$ ) for mapping parasite traits and studying parasite populations. In addition, we report new antigens, providing urgently needed vaccine candidates for disease control.

Genetic mapping using genetic crosses is a powerful approach for identifying genes underlying various traits of parasites ${ }^{7-10}$. However, high costs and laborious procedures have prevented widespread application of genetic crosses for genetic studies in P. falciparum. Association mapping using field isolates is an alternative, but this approach requires typing large numbers of genetic markers and parasite isolates because of the complexity of the parasite population structure and highly variable recombination rates ${ }^{11}$. A genetic map consisting of $\sim 800$ microsatellite markers has been constructed for $P$. falciparum ${ }^{12}$; however, compared with the methods recently developed for large-scale SNP typing ${ }^{13-16}$, microsatellite typing is relatively laborious. A genome-wide map with high-density SNP markers will greatly facilitate our ability to identify important genes underlying parasite traits and address important biological questions pertaining to the genome of $P$. falciparum. Unfortunately, a high-density SNP map is currently available only for chromosome 3 (ref. 11).

We searched for polymorphisms in the $P$. falciparum genome by amplifying and sequencing 3,539 predicted genes or fragments
( $\sim 19 \%$ of each isolate genome; $91 \%$ coding sequence) from four cloned isolates (Dd2, Hb3, D10 and 7G8) (Table 1 and Supplementary Table 1 online). After aligning the DNA sequences to the 3D7 genome sequence ${ }^{1}$, we identified 3,918 well-validated SNPs, giving a genome-wide average of one SNP per $\sim 5.9 \mathrm{~kb}$ DNA (Table 1 and Fig. 1). Of the genes surveyed, approximately half (54.3\%) had one or more SNPs (Supplementary Fig. 1 online); the majority $(\sim 65 \%)$ of SNPs were nonsynonymous (nsSNP; Table 1). The predominance of nsSNPs is probably due to codon bias and high frequencies of nonsynonymous sites in the parasite genome ${ }^{17,18}$. The estimates of the genome-wide average population mutation rate, $4 N \mu$ (Watterson's $\theta$ ), where $N$ is the effective population size and $\mu$ is the per-nucleotide mutation rate, is $5.05 \times 10^{-4}$, and the estimate of average pairwise nucleotide diversity $(\pi)$ is $4.83 \times 10^{-4}$ (Table 1 ), similar to previous reports ${ }^{11}$. Although these diversity values are lower than those from many model organisms, this could be due to our sequencing of mostly coding regions and our high stringency of SNP calling (see Methods).

We resequenced $\sim 45 \mathrm{~kb}$ of DNA covering 183 known SNPs on chromosome 3 from 99 worldwide isolates ${ }^{11}, 108$ of which are common SNPs (minor allele frequency $\geq 0.05$ ). We discovered 185 new SNPs, 29 of which were common SNPs (Supplementary Table 2 online). Because only $\sim 22 \%$ of the $202 \mathrm{~kb}$ sequenced for the five isolates was resequenced for the 99 isolates, we would expect to miss $132(29 / 0.22)$ common SNPs in five isolates. This indicates that our survey of five isolates captures $\sim 45 \%$ of common SNPs (108/ $(132+108)=0.45)$ relative to the worldwide sample of 99 isolates and gives us a frequency of one common SNP per $842 \mathrm{bp}(202 \mathrm{~kb} / 240)$ and a global genome-wide expectation of $>27,000$ common SNPs, as estimated based on SNPs largely from single-copy nontelomeric genes.

Microsatellites are also abundant in the genome, averaging one polymorphic microsatellite per 1.3 genes (Fig. 1 and Table 1). The true frequency of polymorphic microsatellites throughout the genome would probably be much higher if more noncoding regions were assayed. Combining polymorphic microsatellites and SNPs, our data constitute a map with an average of one polymorphic marker per $\sim 3.6 \mathrm{~kb}$ for the P. falciparum genome (Fig. 1), providing a powerful tool for genetic studies of the parasite.

\footnotetext{
${ }^{1}$ Laboratory of Malaria and Vector Research, National Institute of Allergy and Infectious Diseases, National Institutes of Health, Bethesda, Maryland 20892, USA. ${ }^{2}$ Department of Genetics, North Carolina State University, Raleigh, North Carolina 27695-7614, USA. ${ }^{3}$ Department of Statistics, University of Oxford, Oxford OX1 3TG, UK. Correspondence should be addressed to X.-z.S. (xsu@niaid.nih.gov) or P.A. (pawadalla@ncsu.edu).
}

Received 19 June; accepted 2 November; published online 10 December 2006; doi:10.1038/ng1924 
Table 1 Summary of sequenced genes and diversity among five $P$. falciparum isolates

\begin{tabular}{|c|c|c|c|c|c|c|c|c|c|c|c|c|c|}
\hline Chr. & $\begin{array}{c}\text { Bp } \\
\text { sequenced }^{\mathrm{a}}\end{array}$ & $\begin{array}{l}\text { Percentage } \\
\text { sequenced }\end{array}$ & $\begin{array}{c}\text { Genes } \\
\text { sequenced }^{\mathrm{b}}\end{array}$ & $\begin{array}{c}\text { Genes } \\
\text { with SNPs }\end{array}$ & $\begin{array}{l}\text { Number } \\
\text { of SNPs }\end{array}$ & $\begin{array}{l}\text { Genes with } \\
>4 \text { SNPs }\end{array}$ & sSNPs & nsSNPs & ncSNPs & MS & $\theta\left(10^{-4}\right)$ & $\pi\left(10^{-4}\right)$ & Rec. events \\
\hline 1 & 81,256 & 12.6 & 73 (155) & 44 & 120 & 3 & 35 & 81 & 4 & 62 & 6.21 & 6.18 & 18 \\
\hline 2 & 136,980 & 14.5 & $115(224)$ & 70 & 134 & 3 & 48 & 82 & 4 & 70 & 6.71 & 6.52 & 12 \\
\hline 3 & 203,069 & 19.1 & $203(245)$ & 108 & 229 & 7 & 60 & 138 & 31 & 165 & 4.79 & 4.44 & 24 \\
\hline 4 & 128,567 & 10.7 & $114(249)$ & 59 & 120 & 3 & 32 & 86 & 2 & 71 & 4.91 & 5.10 & 16 \\
\hline 5 & 280,845 & 20.9 & $230(330)$ & 118 & 240 & 7 & 68 & 156 & 16 & 172 & 3.98 & 4.7 & 41 \\
\hline 6 & 302,182 & 21.9 & 234 (319) & 130 & 258 & 7 & 78 & 162 & 18 & 198 & 4.36 & 4.21 & 28 \\
\hline 7 & 267,968 & 19.8 & 206 (297) & 139 & 450 & 28 & 147 & 272 & 31 & 186 & 8.27 & 9.55 & 66 \\
\hline 8 & 282,518 & 21.4 & 209 (299) & 125 & 277 & 11 & 80 & 188 & 9 & 191 & 4.84 & 4.51 & 23 \\
\hline 9 & 344,478 & 22.3 & 277 (366) & 153 & 287 & 7 & 89 & 187 & 11 & 237 & 3.98 & 4.85 & 23 \\
\hline 10 & 326,926 & 19.3 & $263(405)$ & 143 & 268 & 6 & 80 & 179 & 9 & 180 & 4.41 & 4.30 & 27 \\
\hline 11 & 387,591 & 19.0 & $311(516)$ & 167 & 365 & 7 & 97 & 244 & 24 & 239 & 4.28 & 4.21 & 47 \\
\hline 12 & 457,373 & 16.6 & 367 (533) & 190 & 343 & 8 & 114 & 213 & 16 & 263 & 3.38 & 3.72 & 30 \\
\hline 13 & 539,419 & 19.2 & 469 (687) & 221 & 407 & 10 & 89 & 293 & 25 & 270 & 3.43 & 4.11 & 36 \\
\hline 14 & 510,063 & 15.5 & $468(776)$ & 253 & 420 & 7 & 133 & 279 & 8 & 244 & 4.12 & 4.33 & 35 \\
\hline Total & $4,249,816$ & 18.6 & $3,539(5,401)$ & 1,920 & 3,918 & 114 & 1,150 & 2,560 & 208 & 2,548 & 4.83 & 5.05 & 426 \\
\hline
\end{tabular}

Chr, chromosome; sSNP, synonymous SNPs; nsSNP, nonsynonymous SNPs; ncSNP, noncoding SNPs; MS polymorphic microsatellites/indels; $\theta$, average population mutation rate; $\pi$, average pairwise nucleotide diversity; rec. events, recombination events detected within each chromosome.

aTotal bp sequenced for each of four isolates. ${ }^{b}$ Numbers in parentheses are the number of predicted genes in 3D7.

을 SNPs are not distributed evenly across chromosomes; rather, some regions have consecutive genes without any SNPs, and other $\checkmark$ DNA segments have consecutive genes with multiple SNPs (Fig. 1). 을 The percentage of genes with SNPs varies from chromosome to chromosome, ranging from $47.1 \%$ (chromosome 13 ) to $67.5 \%$ (chromosome 7) (Table 1). The numbers of SNPs per gene differs more than twofold between chromosomes, averaging 0.85-2.08 SNPs per sequenced gene, with large chromosomes having fewer SNPs per gene (Table 1 and Supplementary Table 1). Indeed, excluding chromosome 7 , there is a negative correlation between chromosome size and the number of SNPs per gene (Supplementary Fig. 2 online). Comparison of the average $\theta$ values from genes (2) at chromosome ends ( $\sim 15 \%$ of the sequenced genes from each chromosome end) with genes in the remainder of the chromosome showed significantly higher $\theta$ values for genes at chromosome ends $(P=0.0001$, Wilcoxon signed rank test; similarly, $P=0.0001$ if we compared and tested ten genes from each end). These results suggest that a generally higher level of polymorphism at chromosome ends may contribute to this negative correlation, because these regions take up a relatively larger proportion of the small chromosomes.

With these genome-wide markers, we estimated the number of recombination events using methods described previously ${ }^{11}$. We detected recombination events at relatively high frequencies (Table 1); they were distributed nonuniformly both within and among chromosomes, clustering in subtelomeric regions, as previously described for a larger sample on chromosome 3 (ref. 11). Understanding the patterns and rates of recombination is of critical importance for genetic studies, particularly association mapping.

Genes encoding surface antigens, cell adhesion molecules and proteins involved in drug interactions are mostly polymorphic (Supplementary Fig. 3 online). The antigen group has a high ratio of nonsynonymous pairwise differences per nonsynonymous site $(\mathrm{pN})$ relative to synonymous pairwise differences per synonymous site $(\mathrm{pS})(\mathrm{pN} / \mathrm{pS}=5.8)$, suggestive of balancing, diversifying or partial directional selection. Additionally, estimates of Tajima's $D$, a measure of the frequency distribution of alleles, across chromosomes also identified some genes that show an excess of

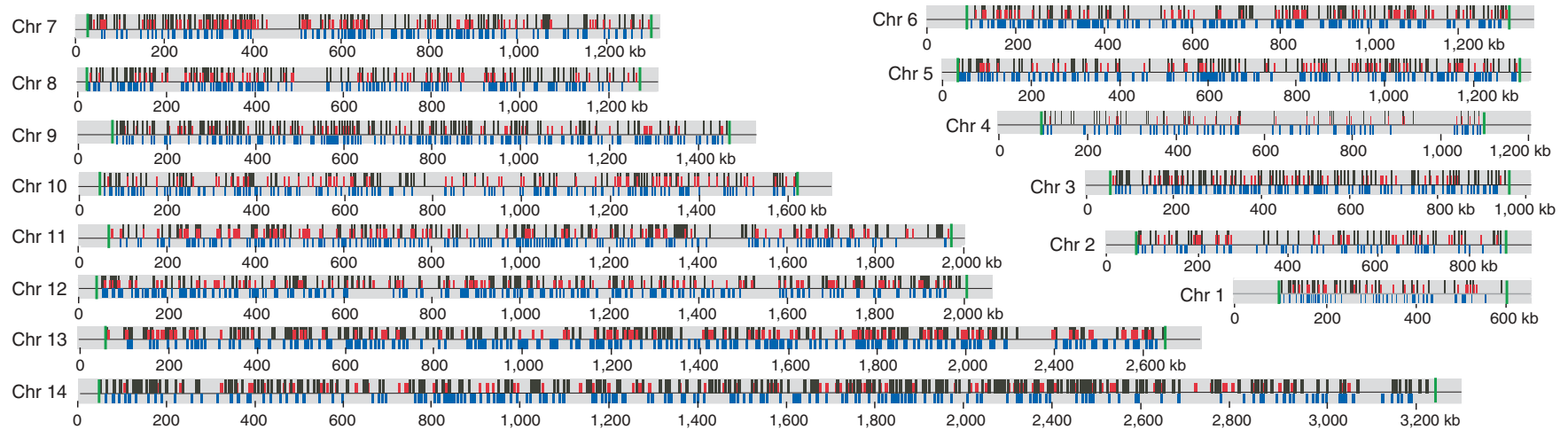

Figure 1 Physical maps showing distribution of polymorphic sites across 14 . falciparum chromosomes. Vertical bars represent SNPs (black, nonsynonymous substitutions; red, synonymous substitutions) or microsatellites (blue, under the horizontal lines). Only one nonsynonymous SNP and one synonymous SNP are presented if there was more than one SNP in a gene (noncoding SNPs were grouped with synonymous SNPs). Most of the chromosomal ends (green vertical bars) were excluded because of gene families such as var, rifin and stevor. 
Table 2 Highly polymorphic genes that are potential immune or drug targets

\begin{tabular}{|c|c|c|c|c|c|}
\hline Gene & Annotation & Number of SNPs & $\theta$ & $\mathrm{TM}$ & Signal peptide \\
\hline PFB0985c & HP & 3 & 0.016941 & 3 & None \\
\hline PF11_0344a,b,c,d & AMA-1 & 48 & 0.012327 & 1 & aa $1-24$ \\
\hline MAL7P1. $6^{\mathrm{b}, \mathrm{d}}$ & HP & 15 & 0.008922 & None & aa $1-21$ \\
\hline MAL7P1.43a & Rifin & 15 & 0.006630 & None & None \\
\hline PFA0655w $w^{a, b}$ & Surfin & 32 & 0.005796 & None & None \\
\hline PF13_0201 a,b & CSP2 & 18 & 0.005729 & None & aa $1-25$ \\
\hline PFB0 $105 c^{b, c}$ & $\mathrm{HP}$ & 7 & 0.005554 & 1 & None \\
\hline MAL7P1.65 & $\mathrm{HP}$ & 14 & 0.004462 & 2 & None \\
\hline PFB0935w $w^{a, b, d}$ & CLAG2 & 9 & 0.004458 & None & aa $1-24$ \\
\hline MAL7P1.66 & $\mathrm{HP}$ & 13 & 0.004315 & None & None \\
\hline MAL7P1.35 & Cell division cycle ATPase & 13 & 0.004254 & None & None \\
\hline MAL7P1.58 & $\mathrm{HP}$ & 6 & 0.004248 & 2 & aa $1-20$ \\
\hline PFI0690c & $\mathrm{HP}$ & 13 & 0.004211 & None & None \\
\hline PFE $1615 c$ & $\mathrm{HP}$ & 3 & 0.004138 & None & None \\
\hline PF08_0005a,b & Tryptophan/threonine-rich antigen & 9 & 0.003702 & 1 & aa $1-21$ \\
\hline PF07_0044 & HP & 3 & 0.003664 & None & None \\
\hline PF14_0445 & HP & 2 & 0.003569 & None & None \\
\hline PFE0815 $w^{b}$ & tRNA pseudouridine synthases & 10 & 0.003550 & None & aa $1-24$ \\
\hline PF11_0109 & $\mathrm{HP}$ & 3 & 0.003478 & None & None \\
\hline PF14_0040d & $\mathrm{HP}$ & 2 & 0.003478 & None & aa $1-19$ \\
\hline PFD1120c & Integral membrane protein & 2 & 0.003466 & 1 & aa $1-28$ \\
\hline MAL13P1.268 & $\mathrm{HP}$ & 7 & 0.003432 & None & aa $1-17$ \\
\hline PFD0310w & Sexual stage-specific protein & 2 & 0.003429 & 1 & aa $1-25$ \\
\hline PFA0665 $w^{a}$ & EBA-like & 8 & 0.003339 & None & None \\
\hline PF14_0759 & $\mathrm{HP}$ & 2 & 0.003265 & 1 & None \\
\hline MAL7P1.59b,d & HP & 5 & 0.003252 & None & None \\
\hline PFL0070c $c^{d}$ & HP & 11 & 0.003212 & None & aa $5-24$ \\
\hline MAL6P1.248 & Histone h3 & 2 & 0.003200 & None & None \\
\hline PF08_0012 & HP & 10 & 0.003198 & None & None \\
\hline PF07_0128 a,b,c & EBA-175 & 10 & 0.003196 & 1 & aa $1-21$ \\
\hline PFE0565w & $\mathrm{HP}$ & 7 & 0.003164 & 1 & aa $1-24$ \\
\hline PFD0110wa,b,d & Reticulocyte binding protein & 8 & 0.003132 & None & aa $1-19$ \\
\hline PF14_0467b,d & $\mathrm{HP}$ & 6 & 0.003006 & 1 & aa $1-24$ \\
\hline PFI0085 $c^{d}$ & HP & 2 & 0.002963 & 2 & None \\
\hline MAL7P1.34d & Cell division cycle ATPase & 4 & 0.002949 & 4 & None \\
\hline PFL1410c & ABC transporter & 9 & 0.002878 & 11 & None \\
\hline PF08_0001 & $\mathrm{HP}$ & 3 & 0.002851 & None & aa $1-17$ \\
\hline PFI0165 $c^{b}$ & DEAD/DEAH box helicase & 9 & 0.002846 & None & None \\
\hline PFD0980w & Holo-acyl-synthase & 7 & 0.002843 & None & None \\
\hline PF14_0019 & $\mathrm{HP}$ & 4 & 0.002832 & None & None \\
\hline PF08_0024 $4^{\mathrm{b}, \mathrm{c}}$ & HP & 5 & 0.002800 & None & aa $1-20$ \\
\hline PF14_0260,d & HP & 7 & 0.002786 & 12 & None \\
\hline PF07_0042 & Lysophospholipase & 9 & 0.002785 & None & None \\
\hline PFI1780w & $\mathrm{HP}$ & 8 & 0.002779 & 1 & None \\
\hline PF07_0124 & HP & 10 & 0.002741 & None & None \\
\hline PF11_0211 d & HP & 7 & 0.002701 & None & None \\
\hline PF13_0275, & $\mathrm{HP}$ & 5 & 0.002667 & 2 & None \\
\hline MAL6P1.231 b,d & Long-chain fatty-acid Co-A ligase & 8 & 0.002663 & 7 & None \\
\hline PF13_0304 ${ }^{\mathrm{b}}$ & Elongation factor 1 alpha & 7 & 0.002646 & None & None \\
\hline PFL2225w & Myosin A tail domain & 2 & 0.002637 & None & None \\
\hline PF14_0014 & HP & 4 & 0.002588 & 1 & aa $1-21$ \\
\hline PF08_0130 & WD repeat protein & 8 & 0.002569 & None & None \\
\hline PF11_0144 & $\mathrm{HP}$ & 5 & 0.002564 & None & None \\
\hline PF13_0198a,b,c & $\mathrm{Rh} 2$ & 9 & 0.002564 & 1 & None \\
\hline PF07_0035d & CG1 & 8 & 0.002540 & None & aa $1-20$ \\
\hline MAL13P1.290 & High mobility-group protein 4 & 2 & 0.002533 & None & None \\
\hline
\end{tabular}

All genes have $\theta$ values greater than the mean $\theta$ (+ 2 s.d.) from 1,920 genes having one or more SNPs. Annotations are based on those in PlasmoDB. HP, hypothetical proteins; $\mathrm{TM}$, number of transmembrane domains in each gene; aa, amino acids (indicates which residues represent a signal peptide).

${ }^{a}$ Known surface proteins and/or antigens. ${ }^{b}$ Genes expressed in a cell-free expression system and detected by antibodies to the His tag. ${ }^{c}$ Genes that encode proteins recognized by pooled human immune sera. ${ }^{\mathrm{d}}$ Genes located in the loci with consecutive polymorphic genes in Supplementary Table 3. 


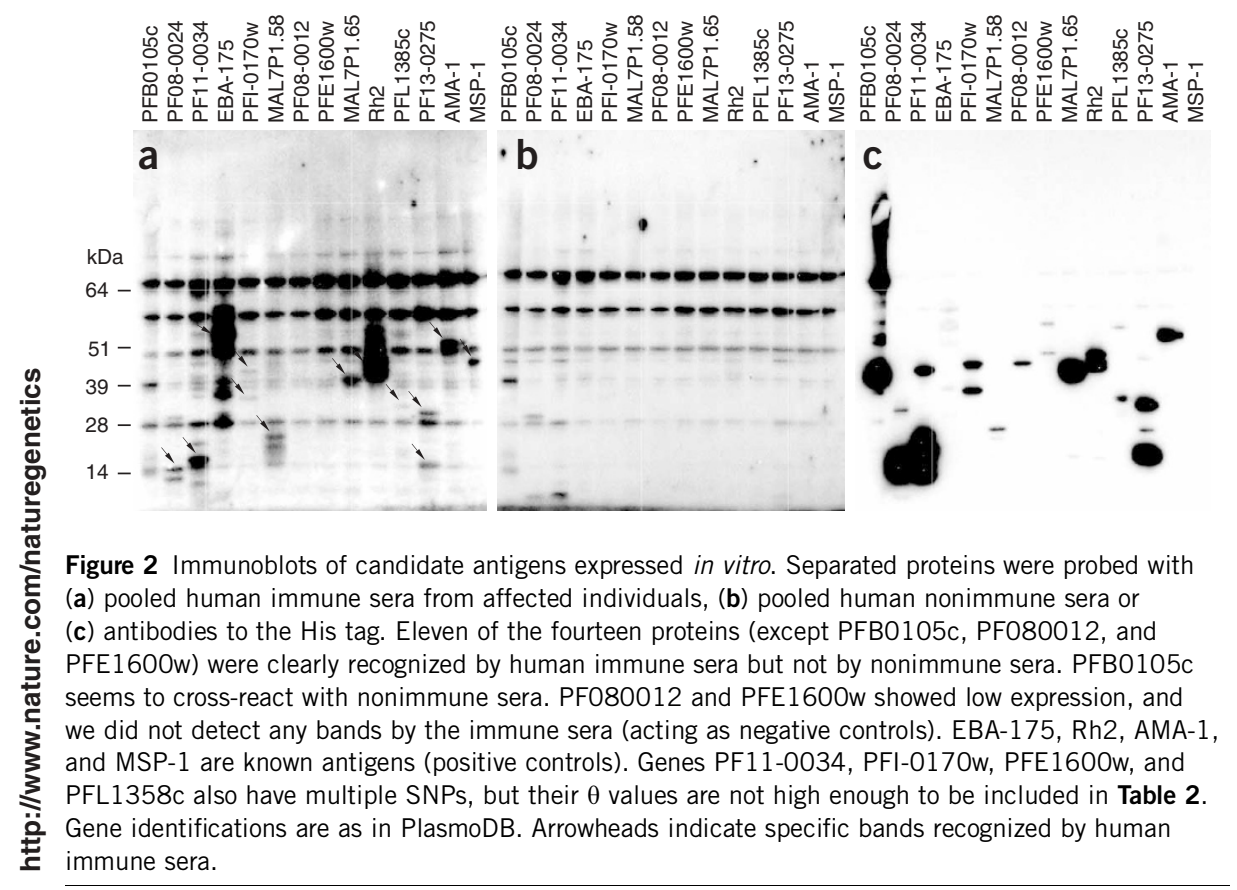

diversity indicative of balancing selection, such as eba-175, which has been shown to be under strong balancing selection (Supplementary Table 1$)^{19}$.

Chromosomal regions flanking many var clusters are more polymorphic than the genome-wide average (Supplementary Fig. 4 and Supplementary Table 1 online). In addition to many subtelomeric var genes, three of the four internal var clusters in the 3D7 genome (on chromosomes 7, 8, and 12) are flanked by five or more consecutive polymorphic genes, with some extending $\sim 100 \mathrm{~kb}$ from the core var cluster, particularly the two clusters on chromo(2) somes 7 and 8 (Supplementary Fig. 4). On one side of the chromosome 7 internal var locus, elevated polymorphism extended over $200 \mathrm{~kb}$ to the chloroquine-resistance transporter gene $(p f c r t)$. Indeed, genes flanking the var loci on chromosomes 7 (25 genes) and 8 (14 genes) have significantly higher $\theta$ values than the average values for their chromosome (Table $1, P<0.0001$ for chromosome 7 and $P<0.001$ for chromosome 8 , Wilcoxon signed rank test). The var genes encode a family of variant antigens called PfEMP1 that are important for immune invasion and disease pathogenesis ${ }^{20-22}$ and that may be under strong balancing selection from the host immune respons $\mathrm{e}^{23}$, thus maintaining more variation than expected under neutrality. Genes flanking var clusters may have correlated evolutionary histories with the var genes, preserving diverse alleles linked to each unique var haplotype. Therefore, a peak of elevated nucleotide diversity surrounding a selected target is one of the signatures of balancing selection ${ }^{24-26}$. Some var clusters, however, do not have obvious elevated polymorphism in the flanking regions, which could be due to the absence of var genes at a specific location in some parasites, or it could be that some var clusters are subject to lesser selective pressures (perhaps expressed less frequently). If this is true, vaccine development based on var genes should probably give emphasis to var genes that are under strong selection.

Signatures of selection can be exploited to identify genes encoding new antigens or drug targets. We searched for genomic regions with consecutive polymorphic genes or peaks of polymorphism (indicative of balancing selection) that may harbor genes encoding antigens. Indeed, approximately $40 \%$ of the 83 loci with five or more consecutive polymorphic genes contain genes encoding known antigens (Supplementary Table 3 and Supplementary Fig. 4 online). These results suggest that most of the parasite antigens are under selection from the host immune system. Further investigation of the other 37 loci (Supplementary Table 3) with unknown genes may lead to some new vaccine candidates. Table 2 lists 56 highly polymorphic genes having $\theta$ values 2 s.d. higher than the mean $\theta$ value for 1,920 genes with one or more SNP. Although more than half of these genes encode proteins of unknown function, $\sim 18 \%$ of them (10) are known antigens (Table 2). Two genes encode proteins involved in lipid metabolism, one of which is a known drug target in other organisms ${ }^{27}$. According to the annotation in PlasmoDB $^{28}$, 32 of these genes $(57 \%)$ have one or more predicted transmembrane domains $(38 \%)$ and/or a signal peptide (36\%), whereas only approximately $11 \%$ and $31 \%$ of the genes in the genome have a predicted signal peptide or transmembrane domain, respectively. The higher proportion of proteins with signal peptides and transmembrane domains suggests potential membrane and/or surface localization that may be recognized by the host immune system.

We next expressed the 56 genes in Table 2 plus 52 genes that have five or more SNPs and encode a predicted signal peptide and/or transmembrane domains (Supplementary Table 4 online) using an E. coli cell-free rapid expression system. Expression of proteins was verified via protein blot using antibodies to the His tags incorporated into the $\mathrm{C}$ terminus of the expressed proteins and detected using pooled human immune sera. Eleven of the 65 expressed proteins were recognized by pooled human immune sera but not by pooled nonimmune sera (Fig. 2); seven of these represented previously unknown antigens that require further evaluation as potential vaccine candidates.

This study identifies thousands of well-validated SNPs and polymorphic microsatellites for mapping genes that may be important in drug resistance, parasite development and disease pathogenesis. Developing high-density markers and high-throughput methods for genotyping large numbers of parasites is critical for mapping genes associated with malaria phenotypes, particularly in high-transmission populations where limited linkage disequilibrium exists. A highthroughput array-based genotyping method is being developed for use in the malaria community. The genome-wide data also show that the $P$. falciparum genome is highly polymorphic, with at least one polymorphic site per $0.5 \mathrm{~kb}$ in only five isolates. Additionally, this work shows that a genome-wide survey for polymorphisms and signatures of selections is a valuable approach for identifying antigens, which should lead to the identification of many new antigens as potential vaccine targets. Our study also suggests that different var clusters may be under variable immune selective pressures that should be taken into consideration when designing a var-based vaccine. Further characterization of the proteins encoded by these genes may lead to new vaccines, which are urgently needed to combat this deadly disease. 


\section{METHODS}

Parasites and DNA amplification and sequencing. DNA sequences of the 3D7 parasite were downloaded from PlasmoDB. Primers for PCR (Supplementary Table 1) and DNA sequencing were designed from predicted ORFs larger than $400 \mathrm{bp}$ ( $\sim 1.5 \mathrm{~kb}$ was sequenced for large genes, excluding the well-known gene families var, stevor and rifin) using a proprietary primer selection software (Visual Basic script). Primers for sequencing both strands of DNA (18-25 bp, with four to seven G/Cs and spaced $\sim 400$ bp apart) were automatically selected and commercially synthesized. Genomic DNA from cultured parasites Dd2 (Thailand), HB3 (Honduras), 7G8 (Brazil) and D10 (Papua New Guinea) of were amplified and sequenced as described previously ${ }^{18}$. Direct sequencing of PCR products eliminates artificial polymorphism frequently introduced when cloning AT-rich DNA into bacteria.

Sequence alignment and analysis. A Java package was written to process raw sequence data, including trimming and aligning DNA sequences using Phred/ Phrap and Sequencher 4.5 (GeneCodes). The 3D7 genomic sequence and the corresponding annotated coding sequences from PlasmoDB 5.0 (both sets of chromosomal flat files dated 2002 and 2005) provided the gene annotations, including annotation of coding and noncoding regions and gene ontology classifications. The program also mapped alignment files to the 3D7 genome and characterized any variation found in the alignment as SNPs or indels or microsatellites. A set of scripts was then used to calculate summary statistics of diversity $(\theta, \pi$ and Tajima's $D$ values). All SNPs and microsatellites were confirmed by visually inspecting chromatogram traces of all potential poly을 morphisms. All alignments of indels and microsatellites were manually adjusted to minimize mismatches and size polymorphism. SNPs in repetitive regions were not called, because misalignments may create artificial SNPs. The number of of recombination events throughout the genome was estimated using the nonparametric methodology of ref. 29 as described previously ${ }^{11}$.

Protein expression and blotting. Cell-free expression of proteins was performed using a rapid translation system (Roche Diagnostics) according to the manufacturer's instruction. Proteins expressed in $50 \mu \mathrm{l}$ of an E. coli cell-free expression system were enriched using paramagnetic precharged nickel particles (Promega), separated on $4 \%-12 \%$ polyacrylamide gels, transferred to PVDF membrane and detected using antibodies to the His tag or pooled human antisera from villagers of Mali.

Accession codes. SNPs have been deposited at NCBI dbSNP database (accession codes 65654288-65658180) and also at PlasmoDB (v5.2).

URLs. PlasmoDB: http://www.plasmodb.org/. Phred/Phrap: http://www. phrap.org/.

Note: Supplementary information is available on the Nature Genetics website.

\section{ACKNOWLEDGMENTS}

We thank C. Long and R. Fairhurst for pooled immune human sera and National Institute of Allergy and Infectious Disease (NIAID) intramural editor B.R. Marshall for assistance. This work was supported by the Division of Intramural Research of the NIAID as well as by the US National Institutes of Health, the National Academies Keck Genome Initiative and the Human Frontiers in Science Program (P.A.).

\section{AUTHOR CONTRIBUTIONS}

J.M.: DNA amplification, sequencing and data analysis; J.D.: DNA amplification and sequencing; K.S.: primer design; K.M.M. and J.K.: software and database development and data analysis; G.A.T.M.: manuscript preparation; P.A.: software and database development, data analysis and manuscript preparation; X-z.S.: project design, data analysis and manuscript preparation.

\section{COMPETING INTERESTS STATEMENT}

The authors declare that they have no competing financial interests.
Published online at http://www.nature.com/naturegenetics

Reprints and permissions information is available online at http://npg.nature.com/ reprintsandpermissions/

1. Gardner, M.J. et al. Genome sequence of the human malaria parasite Plasmodium falciparum. Nature 419, 498-511 (2002).

2. Polley, S.D. \& Conway, D.J. Strong diversifying selection on domains of the Plasmodium falciparum apical membrane antigen 1 gene. Genetics 158, 1505-1512 (2001).

3. Conway, D.J. et al. A principal target of human immunity to malaria identified by molecular population genetic and immunological analyses. Nat. Med. 6, 689-692 (2000).

4. Volkman, S.K. et al. Excess polymorphisms in genes for membrane proteins in Plasmodium falciparum. Science 298, 216-218 (2002).

5. Wootton, J.C. et al. Genetic diversity and chloroquine selective sweeps in Plasmodium falciparum. Nature 418, 320-323 (2002).

6. Roper, C. et al. Intercontinental spread of pyrimethamine-resistant malaria. Science 305, 1124 (2004).

7. Wellems, T.E., Walker-Jonah, A. \& Panton, L.J. Genetic mapping of the chloroquineresistance locus on Plasmodium falciparum chromosome 7. Proc. Natl. Acad. Sci. USA 88, 3382-3386 (1991).

8. Su, X.-Z., Kirkman, L.A., Fujioka, H. \& Wellems, T.E. Complex polymorphisms in an approximately $330 \mathrm{kDa}$ protein are linked to chloroquine-resistant $P$. falciparum in Southeast Asia and Africa. Cell 91, 593-603 (1997).

9. Vaidya, A.B. et al. A genetic locus on Plasmodium falciparum chromosome 12 linked to a defect in mosquito-infectivity and male gametogenesis. Mol. Biochem. Parasitol. 69, 65-71 (1995).

10. Wang, P., Read, M., Sims, P.F. \& Hyde, J.E. Sulfadoxine resistance in the human malaria parasite Plasmodium falciparum is determined by mutations in dihydropteroate synthetase and an additional factor associated with folate utilization. Mol. Microbiol. 23, 979-986 (1997).

11. Mu, J. et al. Recombination hotspots and population structure in Plasmodium falciparum. PLoS Biol. 3, e335 (2005).

12. Su, X.-z. et al. A genetic map and recombination parameters of the human malaria parasite Plasmodium falciparum. Science 286, 1351-1353 (1999).

13. Lindblad-Toh, K. et al. Large-scale discovery and genotyping of single-nucleotide polymorphisms in the mouse. Nat. Genet. 24, 381-386 (2000).

14. Kennedy, G.C. et al. Large-scale genotyping of complex DNA. Nat. Biotechnol. 21, 1233-1237 (2003).

15. Hardenbol, P. et al. Highly multiplexed molecular inversion probe genotyping: over 10,000 targeted SNPs genotyped in a single tube assay. Genome Res. 15, 269-275 (2005).

16. Gunderson, K.L., Steemers, F.J., Lee, G., Mendoza, L.G. \& Chee, M.S. A genome-wide scalable SNP genotyping assay using microarray technology. Nat. Genet. 37, 549-554 (2005).

17. Hey, J. Parasite populations: the puzzle of Plasmodium. Curr. Biol. 9, R565-R567 (1999).

18. Mu, J. et al. Chromosome-wide SNPs reveal an ancient origin for Plasmodium falciparum. Nature 418, 323-326 (2002).

19. Baum, J., Thomas, A.W. \& Conway, D.J. Evidence for diversifying selection on erythrocyte-binding antigens of Plasmodium falciparum and P. vivax. Genetics 163, 1327-1336 (2003).

20. Baruch, D.I. et al. Cloning the $P$. falciparum gene encoding PfEMP1, a malarial variant antigen and adherence receptor on the surface of parasitized human erythrocytes. Cell 82, 77-87 (1995).

21. Su, X.-z. et al. The large diverse gene family var encodes proteins involved in cytoadherence and antigenic variation of Plasmodium falciparum-infected erythrocytes. Cell 82, 89-100 (1995).

22. Smith, J.D. et al. Switches in expression of Plasmodium falciparum var genes correlate with changes in antigenic and cytoadherent phenotypes of infected erythrocytes. Cell 82, 101-110 (1995).

23. Trimnell, A. et al. Global genetic diversity and evolution of var genes associated with placental and severe childhood malaria. Mol. Biochem. Parasitol. 148, 169-180 (2006).

24. Hudson, R.R. \& Kaplan, N.L. The coalescent process in models with selection and recombination. Genetics 120, 831-840 (1988).

25. Nordborg, M., Charlesworth, B. \& Charlesworth, D. Increased levels of polymorphism surrounding selectively maintained sites in highly selfing species. Proc. R. Soc. Lond. B 263, 1033-1039 (1996).

26. Charlesworth, D. Balancing selection and its effects on sequences in nearby genome regions. PLoS Genet. 2, e64 (2006).

27. Sato, S. \& Wilson, R.J. The plastid of Plasmodium spp.: a target for inhibitors. Curr. Top. Microbiol. Immunol. 295, 251-273 (2005).

28. Kissinger, J.C. et al. The Plasmodium genome database. Nature 419, 490-492 (2002).

29. Myers, S.R. \& Griffiths, R.C. Bounds on the minimum number of recombination events in a sample history. Genetics 163, 375-394 (2003). 